medications, pulmonary outcomes assessed with repeated pulmonary function tests (PFTs) and chest HRCT and complications in a 1-year follow-up period were documented for each patient. Univariate models were performed in order to identify determinants of infection and clinically significant difference in PFTs (defined as change of $\geq 10 \%$ in FVC and/or $\geq 15 \%$ in DLCO).

Results: The mean age at the time of IPAF diagnosis was $63.2( \pm 11)$ years and $62 \%$ of the patients were female. The most common clinical features included in the IPAF criteria were arthritis (82\%) and Raynaud's phenomenon (26\%). A morbilliform and/or polymorphic rash of the face, neck and extremities (not included in the IPAF criteria) was noted in $54 \%$ of patients. ANA (59\%) and anti-Ro (21\%) were the most common auto-antibodies. Non-specific Interstitial Pneumonia (NSIP) was the most prevalent radiological pattern (61.5\%) as shown in table 1. Treatment comprised corticosteroids and immunosuppressants including hydroxychloroquine, methotrexate, azathioprine, mycophenolate and cyclophosphamide. PFTs following treatment at 6 and 12 months from baseline showed a trend of improvement (Table 2, p> 0.05). At 1 year from baseline, $20.5 \%$ of patients showed a clinically significant deterioration while $25 \%$ had a clinically significant improvement. Infections were observed in $23.1 \%$ of patients during the first semester and in $12.8 \%$ during the second semester of the follow-up period. All were respiratory tract infections and two patients $(5.1 \%)$ required hospitalization. All infections occurred in patients with non-UIP pattern $(p=0.02)$ which might be attributed to higher doses of corticosteroids used in these patients (mean initial prednisolone dose $=27( \pm 18) \mathrm{mg} / \mathrm{d}$ in patients with non-UIP pattern versus 17 $( \pm 16) \mathrm{mg} / \mathrm{d}$ in patients with UIP pattern, $\mathrm{p}=0.4$ )

Table 1. Prevalence of HRCT patterns in 39 patients.

\begin{tabular}{lc}
\hline Radiological pattern & No (\%) \\
\hline NSIP & $24(61,5 \%)$ \\
OP & $2(5,1 \%)$ \\
NSIP with OP overlap & $2(5,1 \%)$ \\
LIP & $1(2,6 \%)$ \\
UIP & $7(18 \%)$ \\
NSIP and UIP & $3(7,7 \%)$ \\
\hline
\end{tabular}

NSIP: Non-specific Interstitial Pneumonia, OP: Organizing Pneumonia, LIP: Lymphocytic Interstitial Pneumonia, UIP: Usual Interstitial Pneumonia.

Table 2. PFTs at baseline, 6 and 12 months.

\begin{tabular}{lcccc}
\hline PFTs $(\%$ of predicted value \pm SD) & Baseline & 6 months & 12 months & P value \\
\hline FVC & $79 \%( \pm 19 \%)$ & $82 \%( \pm 18 \%)$ & $84 \%( \pm 17 \%)$ & $\mathrm{ns}$ \\
DLCO & $49 \%( \pm 16 \%)$ & $52 \%( \pm 17 \%)$ & $53 \%( \pm 17 \%)$ & $\mathrm{ns}$
\end{tabular}

Conclusion: Rash is a common feature in IPAF and may be considered for inclusion into IPAF criteria. A trend of improvement in PFTs and a significant risk of respiratory tract infections mainly in the first semester of treatment and in patients with non-UIP radiological pattern were observed. Larger prospective studies are warranted in order to elucidate IPAF's prognosis and to identify effective management approaches.

References:

[1] Fischer A, et al. An official European Respiratory Society/American Thoracic

Society research statement: interstitial pneumonia with autoimmune features. Eur Respir J 2015; 46: 976-987.

Disclosure of Interests: Maria Karampeli: None declared, Konstantinos Thomas: None declared, Dimitrios Tseronis: None declared, Michail Aggelakos: None declared, Dimitra Kassara: None declared, Katerina Havatza: None declared, Sofia Flouda: None declared, Dionysis Nikolopoulos: None declared, Antigoni Pieta: None declared, Vasiliki Tzavara: None declared, Pelagia Katsimbri: None declared, Dimitrios Boumpas Grant/research support from: Unrestricted grant support from various pharmaceutical companies, Theofanis Karageorgas: None declared

DOI: 10.1136/annrheumdis-2020-eular.2753

\section{AB1217 THE EFFECT OF PROPHYLACTIC DOSE OF TRIMETHOPRIM-SULFAMETHOXAZOLE ON SERUM CREATININE IN JAPANESE PATIENTS WITH CONNECTIVE TISSUE DISEASES}

R. Kawato ${ }^{1}$, R. Rokutanda ${ }^{2}$, M. Okada ${ }^{3} .{ }^{1}$ Juntendo University Graduate School of Medicine, Department of Rheumatology, Tokyo, Japan; ${ }^{2}$ Kameda Medical Center, Department of Rheumatology, Chiba, Japan; ${ }^{3}$ St. Luke's International Hospital, Immuno-Rheumatology Center, Tokyo, Japan

Background: Trimethoprim-sulfamethoxazole (TMP/SMX) is an effective antibiotic for prevention of pneumocystis pneumonia (PCP) in immunocompromised patients with various connective tissue diseases (CTD). ${ }^{1)}$ At the normal dose of TMP/SMX, trimethoprim (TMP) component inhibits tubular creatinine secretion, leading to a rapid, but ultimately reversible, increase in serum creatinine. ${ }^{2)} \mathrm{How}$ ever, at prophylactic dose, the effect of TMP/SMX on creatinine in patients with CTD is not clear.

Objectives: We conducted this study to evaluate the effect of prophylactic dose of TMP/SMX on serum creatinine in patients with CTD.

Methods: This was a retrospective cohort study in which all patients with CTD treated with prophylactic dose of TMP/SMX during the period between 2004 and 2018, were included, while patients with acute kidney injury due to other causes, were excluded. Retrospective medical chart review was performed to collect the following data, from the baseline through 12 weeks: baseline patient characteristics, serum creatinine $(\mathrm{SCr})$, creatinine clearance $(\mathrm{CCr})$, urine test, serum electrolytes, and level of SCr elevation after initiation of prophylactic dose of TMP/SMX from baseline, within 12 weeks. Using single and multiple regression analyses, we explored the risk factors that affected the $\mathrm{SCr}$ elevation value. Results: A total of 272 patients, comprising 186 females, at an average age of $56 \pm 18$ years, were included in the present study. Based on the medical char review, this cohort was scored under the following categories: rheumatoid arthritis (RA) $n=78$, systemic lupus erythematosus (SLE) $n=76$, Vasculitis $n=39$, polymyalgia rheumatica/ giant cell arteritis (PMR/GCA) $n=29$, systemic sclerosis (SSc) $n=32$, polymyositis/dermatomyositis $(P M / D M) n=10$, mixed CTD (MCTD) $n=5$, and others $n=56$. Their average baseline creatinine level before treatment was $0.67 \pm$ $0.25 \mathrm{mg} / \mathrm{dL}$. They were administered with a mean dose of TMP comprising 99.2 $\pm 34.4 \mathrm{mg} /$ day, which elevated the mean SCr level by $0.07 \pm 0.12 \mathrm{mg} / \mathrm{dL}$. Approximately $85 \%$ of the patients continued with the TMP/SMX treatment for 12 weeks, and only $5(2 \%)$ showed creatinine elevation by more than $0.3 \mathrm{mg} / \mathrm{dL}$. They were also administered with prednisolone at an average dose of $36.9 \pm 92.3 \mathrm{mg} / \mathrm{day}$. For multiple regression analyses, the following variables were included: age baseline CCr, use of loop diuretics, use of spironolactone, dose of TMP/SMX use of non-steroidal anti-inflammatory drugs, and past history of diabetes mellitus. Baseline $\mathrm{CCr}$ and advanced age were independent risk factors. Regression coefficients and $p$ values for age and CCr were $0.0018(95 \% \mathrm{Cl} ; 0.00083$ $-0.0027)$ and $p=0.00028$; and $0.0007(95 \% \mathrm{Cl} ; 0.0003-0.0010)$ and $\mathrm{p}=0.00038$, respectively.

Conclusion: In this study, we demonstrated that prophylactic dose of TMP/ $\mathrm{SMX}$ elevates $\mathrm{SCr}$ by $0.07 \pm 0.12 \mathrm{mg} / \mathrm{dL}$ on an average. Prophylactic dose of TMP/SMX rarely elevated the creatinine level significantly. Thus, based on our findings, other causes of renal impairment may be considered, if the patients administered with low-dose TMP/SMX show creatinine elevation by more than $0.3 \mathrm{mg} / \mathrm{dL}$.

References:

[1] Prophylactic effect of trimethoprim-sulfamethoxazole for pneumocystis pneumonia in patients with rheumatic diseases exposed to prolonged high-dose glucocorticoids Park JW, et al. Ann Rheum Dis 2018;77:644-649.

[2] Factors contributing to increases in serum creatinine following treatment with a sulfamethoxazole-trimethoprim combination product: retrospective analysis of Japanese patients with normal renal function. Muramori, et al. YAKUGAKU ZASSHI 133(5) 587-595 (2013)

Disclosure of Interests: None declared

DOI: 10.1136/annrheumdis-2020-eular.397

\section{\begin{tabular}{|l|l}
\hline AB1218 MATERNAL-FETAL MORBIDITY AND MORTALITY \\
\hline
\end{tabular} AMONG PREGNANT PATIENTS WITH RHEUMATIC DISEASES IN THE CLINIC OF PREGNANCY AND ARTHRITIS OF THE SALVADORAN SOCIAL SECURITY INSTITUTE}

R. I. López ${ }^{1}$, R. Montufar ${ }^{1}$, D. Alpizar-Rodriguez ${ }^{2} .{ }^{1}$ Instituto Salvadoreño del Seguro Social, San Salvador, El Salvador; ${ }^{2}$ Colegio Mexicano de Reumatología, Ciudad de México, Mexico

Background: Connective tissue diseases are more frequent among women in childbearing age. In the past, it was not recommended for these women to get pregnant because of the risk of adverse pregnancy outcomes.

Objectives: The aim of this study is to compare the morbidity and mortality between pregnant patients with rheumatic diseases and pregnant patients without rheumatic diseases in El Salvador.

Methods: A case control study was conducted at the Clinic of Pregnancy and Arthritis and the $1^{\circ}$ de Mayo Hospital, both centers belong to the Salvadoran Social Security Institute. Clinical files were reviewed retrospectively and some of the data were collected during the clinical visit to each center. We included pregnant patients with rheumatic diseases of the Clinic of Pregnancy and Arthritis; the control group was randomly selected with a ratio 1:1 within pregnant patients without rheumatic diseases of the $1^{\circ}$ de Mayo Hospital, between January 2016 and June 2019. A complication was defined as any event that required hospital admission. We used logistic regression to analyze univariable and multivariable associations to compare pregnancy outcomes between groups.

Results: During the study period, 230 patients were randomly included in both, the study group and the control group, with a ratio 1:1. The mean age for the study group was 32 (27-35) years, which was relatively older than the control 
group, 28 (24-32) years. The mean pregnancy among both groups was 2 per patient. The most common rheumatic diseases in the study group were Rheumatoid arthritis (39.1\%), Antiphospholipid Syndrome (31.3\%) and Generalized Lupus Erythematosus (20\%). The average visit to the Rheumatologist during pregnancy in the study group was 3.2. The visits started at a mean of $15.2+7.6$ weeks of gestational age.

Thirty three point nine percent $(33.9 \%)$ of the study group presented complications during pregnancy (vs $20.9 \%$ ). Among the patients who presented complications, 9 of them $(7.8 \%)$ had more than one complication during pregnancy. There were $44.3 \%$ cesarean deliveries in the study group compared with $22.6 \%$ in the control group, OR $2.1(1.5-4.8) \mathrm{p}=0.001$. There were $16 \%$ of preterm deliveries between 34-37 weeks of gestational age in the study group compared to $10 \%$ in the control group, OR $2.2(0.9-4.8) p=0.063$. Preterm deliveries before 34 weeks of gestational age in the study group were $18 \%$ compared to $3 \%$ in the control group, OR 6.9 (2.3-20-9) p=0.001. There were 6 cases of spontaneous abortion in the study group and none in the control group. Thirty four percent $(34 \%)$ of cases with low birth weight $(<2.5 \mathrm{~kg})$ were found in the study group compared to $14 \%$ in the control group, OR 3.4 (1.6-6.1) $p=0.001$.

Conclusion: The most common rheumatic diseases among pregnant patients were Rheumatoid Arthritis, Antiphospholipid syndrome and Generalized Lupus Erythematosus.

Having a rheumatic disease was more associated with cesarean delivery, preterm delivery between 34 and 37 weeks of gestational age, and low birth weight. References:

[1] M. Gayed, C. Gordon. Pregnancy and rheumatic diseases. Rheumatology 2007;46:1634-1640.

[2] Y A de Man, et al. Annals of Rheumatic Diseases. 2008. 10.1136

Disclosure of Interests: None declared

DOI: 10.1136/annrheumdis-2020-eular.5051

\section{$\mathrm{AB} 1219$ \\ ADHERENCE TO THE MEDITERRANEAN DIET IN PATIENTS WITH RHEUMATOID ARTHRITIS AND OSTEOARTHRITIS, MULTICENTER STUDY}

I. Morales-Ivorra ${ }^{1}$, D. Grados Canovas ${ }^{1}$, A. Rozadilla ${ }^{2}$, J. M. Nolla ${ }^{2}$, B. Busque ${ }^{1}$, J. Bové ${ }^{1}$, D. Madrid ${ }^{1}$, L. Valencia Muntalà ${ }^{2}$, M. Romera-Baurés ${ }^{2} .{ }^{1}$ Hospital Igualada, Igualada, Spain; ${ }^{2}$ Hospital Universitari de Bellvitge, Hospitalet de Llobregat, Spain

Background: The Mediterranean diet (MD) has proven beneficial in a large number of chronic diseases. The relationship between the MD and rheumatic diseases is complex and there are few studies that have studied this relationship. These show that there could be a positive association between adherence to the Mediterranean diet (MD-A) and a lower prevalence of OA. In the case of RA, it has been proposed that the MD could reduce pain and improve functionality.

Objectives: To determine the MD-A diet of patients with RA and OA, and compare it with that of healthy subjects.

Methods: Multicenter, cross-sectional, observational study. Patients who attend the rheumatology outpatient and meet the ACR / EULAR 2010 criteria for RA and ACR for OA of hands, knees or hips are included in the study. The healthy are recruited among health personnel and companions of patients who do not live in the same address as the patient. The study is being carried out in the rheumatology consultations of two Hospitals and an outpatient center with specialized care. All participants have answered a survey of 14 questions (MEDAS14), based on the Predimed study, which assesses MD-A. Fisher's exact test and the Mann-Whitney $U$ test have been used to assess statistical significance. The study was approved by the Clinical Ethics and Research Committee of the centers.

Results: There have been 279 surveys (132 RA, 82 OA and 65 healthy). The MD-A in patients with RA is lower than in healthy (6.26 vs. $7.15, p<0.05)$. Patients with $\mathrm{OA}$ also have less adherence to the MD than healthy ones but this difference is not statistically significant ( 6.85 vs. $7.15, p>0.05)$. The proportion of patients with RA and OA who consume 2 or more servings of vegetables daily is lower than that of healthy subjects (RA $20 \%$; OA $13 \%$ and healthy $34 \%, p<0.05$ ). The proportion of RA and OA that eats more than 3 weekly servings of nuts compared to healthy is also lower (RA $21 \%$, OA $17 \%$, healthy $35 \% \mathrm{p}<0.05$ ). The proportion of RA and $\mathrm{OA}$ that consume less than 1 serving of butter is lower than that of healthy (RA $86 \%$; OA $82 \%$ and $98 \%, p<0.05)$. The proportion of RA that consumes 3 or more servings of legumes per week is lower than healthy ( $23 \%$ vs $40 \%, p<0.05)$. These differences between the OA group and healthy are not appreciated. The consumption of more than three pieces of fruit daily is more frequent in $O A$ than in healthy ones ( $45 \%$ vs $26 \%$, $p<0.05$ ).

Conclusion: The MD-A diet quantified by MEDAS-14 in subjects with RA and $\mathrm{OA}$ is lower than in healthy subjects, being significant in RA. Patients with RA and $O A$ eat less vegetables and nuts but the intake of butter is higher. The RA group consumes less legumes than healthy ones. Patients with OA eat more fruit than healthy ones, this is the only food in the MD valued by MEDAS-14 that is consumed in a lower proportion in healthy ones. Longitudinal intervention studies are necessary to assess whether the differences observed in this study have any causal relationship.

\section{References:}

[1] Estruch R, Ros E, Salas-Salvadó J, Covas M-I, Corella D, Arós F, et al. Primary prevention of cardiovascular disease with a Mediterranean diet. N Engl J Med. 2013;368(14):1279-90.

[2] Trichopoulou A, Martínez-González MA, Tong TY, Forouhi NG, Khandelwal S, Prabhakaran D, et al. Definitions and potential health benefits of the Mediterranean diet: views from experts around the world. BMC Med. 2014;12:112.

[3] Morales-Ivorra I, Romera-Baures M, Roman-Viñas B, Serra-Majem L. Osteoarthritis and the Mediterranean Diet: A Systematic Review. Nutrients. 2018;10(8) pii: E1030. doi: 10.3390/nu10081030.

[4] Forsyth C, Kouvari M, D'Cunha NM, Georgousopoulou EN, Panagiotakos DB Mellor DD, et al. The effects of the Mediterranean diet on rheumatoid arthritis prevention and treatment: a systematic review of human prospective studies. Rheumatol Int. 2018 May;38(5):737-47.

Disclosure of Interests: None declared

DOI: 10.1136/annrheumdis-2020-eular.3336

\section{AB1220 DO ANTIBODIES HAVE DIFFERENTIAL EFFECT ON RADIOGRAPHIC PROGRESSION IN RA?}

A. Minocha ${ }^{1}$, S. Kukran ${ }^{1}$, P. Yee ${ }^{1}$, M. K. Nisar ${ }^{1} .{ }^{1}$ Luton and Dunstable University Hospital, Luton, United Kingdom

Background: The presence of bony erosions in patients with RA is a marker of disease severity and once present they are largely irreversible. Previous studies have shown that those who are RF positive have more erosions than those who are RF negative. Likewise, ACPA is associated with increased bone loss and rapid erosive changes.

Objectives: The aim of our study was to determine which of these two antibodies is more strongly associated with erosive disease and whether being double +ve confers higher risk than single positive antibody status.

Methods: A retrospective study of 774 patients diagnosed with RA at a large university teaching hospital during the period of January 1981 and December 2018. Clinical records were reviewed to determine antibody status, date of diagnosis, duration of symptoms, DAS-28, age, ethnicity and whether the 1987 Rheumatoid Arthritis criteria was met. The presence of radiographic erosions at diagnosis were determined from reports of plain film radiographs of hands and feet of all patients. Chi square model was utilised to ascertain if there was a significant relationship among the four groups. Mann Whitney two-tailed $U$ test was employed to determine the significance of relationship between the double negative group and other arms for all variables including disease duration and delta change in DAS28. Significance level was predefined at 0.05

Results: All patients fulfilled the 2010 ACR/EULAR criteria. 240 (31\%) patients were male and 534 (69\%) were female with an age range of 17 to 90 years for the cohort. The duration of symptoms ranged for the patient cohort from 0.5 to 250 months with a median of 6 months while the duration of disease ranged from 4 to 455 months with a median of 49 months. DAS 28 ranged from 1.19 to 8.4 with a median of $4.4 .367(47 \%)$ were positive for both RF and ACPAs, 87 (11\%) were positive for RF alone, 66 (9\%) were positive for ACPA alone and 254 (33\%) were antibody negative.

There were a total of 127 patients with erosions at the time of diagnosis. Patients in the double positive group had a significantly higher $(p=0.003)$ erosion burden compared to the double negative group i.e. $21.5 \%$ in $\mathrm{RF}+/ \mathrm{ACPA}+$ versus $11.0 \%$ in RF-/ACPA- group. The erosion burdens in RF+/ACPA- and RF-/ACPA+ groups were $13.7 \%$ and $12.1 \%$ respectively.

Conclusion: This is the first real world study to demonstrate the radiographic erosive burden in the RA cohort with respect to RF/ACPA status. Our results show that patients $R F+/ A C P A+$ have nearly two-fold higher incidence of radiographic erosions than patients who are RF-/ACPA-. Patients with single antibody positivity have a trend towards higher erosive burden compared to the double antibody negative group however it's the combination of two antibodies which is strongly associated with erosive disease.

Disclosure of Interests: Amal Minocha: None declared, Sebi Kukran: None declared, Philip Yee: None declared, Muhammad Khurram Nisar Grant/research support from: Muhammad Nisar undertakes clinical trials and received support (including attendance at conferences, speaker fees and honoraria) from Roche, Chugai, MSD, Abbvie, Pfizer, BMS, Celgene, Novartis and UCB

Consultant of: Muhammad Nisar undertakes clinical trials and received support (including attendance at conferences, speaker fees and honoraria) from Roche, Chugai, MSD, Abbvie, Pfizer, BMS, Celgene, Novartis and UCB

Speakers bureau: Muhammad Nisar undertakes clinical trials and received support (including attendance at conferences, speaker fees and honoraria) from Roche, Chugai, MSD, Abbvie, Pfizer, BMS, Celgene, Novartis and UCB DOI: 10.1136/annrheumdis-2020-eular.1963

\section{$\mathrm{AB} 1221$} POPULATION WIDE STUDY OF MORTALITY IN ANCAASSOCIATED VASCULITIS IN WESTERN AUSTRALIA FROM 2000 TO 2014

W. Taylor ${ }^{1}$, W. Raymond ${ }^{1}$, H. Keen ${ }^{2,3}$, C. Inderjeeth ${ }^{3,4}$, D. Preen ${ }^{5}$, J. " Nossent ${ }^{4,6}$ ${ }^{1}$ University of Western Australia, Rheumatology Section, School of Medicine, Crawley, Australia; ${ }^{2}$ Fiona Stanley Hospital, Rheumatology, Murdoch, Australia;

${ }^{3}$ The University of Western Australia, Rheumatology Section, School of 